\title{
Weighted a priori bounds for elliptic operators of Cordes type
}

\section{Loredana Caso ${ }^{*}$, Roberta D'Ambrosio and Maria Transirico}

"Correspondence: lorcaso@unisa.it Department of Mathematics, University of Salerno, via Giovanni Paolo II, 132, Fisciano, SA 84084, Italy

\begin{abstract}
We prove some weighted $W^{2,2}$-a priori bounds for a class of elliptic second order linear differential operators of Cordes type on unbounded domains of $\mathbb{R}^{n}, n \geq 2$.
\end{abstract}

MSC: 35J25; 35B45; 35R05

Keywords: elliptic equations; a priori bounds; weighted spaces

\section{Introduction}

Consider the Dirichlet problem

$$
\left\{\begin{array}{l}
u \in W^{2,2}(\Omega) \cap \stackrel{\circ}{W}^{1,2}(\Omega), \\
L u=f, \quad f \in L^{2}(\Omega)
\end{array}\right.
$$

where $\Omega$ is an open subset of $\mathbb{R}^{n}, n \geq 2$, with a suitable regularity property, and $L$ is an elliptic second order linear differential operator, with measurable coefficients, defined by

$$
L=-\sum_{i, j=1}^{n} a_{i j} \frac{\partial^{2}}{\partial x_{i} \partial x_{j}}+\sum_{i=1}^{n} a_{i} \frac{\partial}{\partial x_{i}}+a .
$$

If $\Omega$ is bounded and the coefficients $a_{i j}$ are continuous, it is well known that problem (1) is uniquely solvable (see, for instance, the classical results in $[1,2]$ ).

In the framework of discontinuous coefficients, special attention is paid to the so-called Cordes condition introduced by HO Cordes in the study of Hölder continuity of solutions of (1) (see [2, 3]). It replaces the hypothesis on the continuity of the $a_{i j}$ 's with the requirement that the eigenvalues of the matrix of the coefficients $A=\left(a_{i j}\right)$ do not scatter too much. The Cordes condition enabled Talenti in [4] to provide the well-posedness of problem (1) for elliptic operators $L$ without the lower order terms $a_{i}, a$, and on bounded domains of $\mathbb{R}^{n}, n \geq 3$. Later on, the author studied the solvability of problem (1) also in the planar case, only assuming the following condition on the leading coefficients:

$$
a_{i j}=a_{j i} \in L^{\infty}(\Omega), \quad i, j=1,2,
$$

together with the boundedness of the lower order terms $a_{i}, a$ of operator $L$ defined in (2) (see [5]).

(c) 2015 Caso et al. This article is distributed under the terms of the Creative Commons Attribution 4.0 International License (http://creativecommons.org/licenses/by/4.0/), which permits unrestricted use, distribution, and reproduction in any medium, provided you give appropriate credit to the original author(s) and the source, provide a link to the Creative Commons license, and indicate if changes were made. 
Next in [6] the above mentioned results in [3, 4] were extended to elliptic equations of type (1) where the operator $L$ of Cordes type has lower order coefficients in suitable Lebesgue spaces. Uniqueness and existence results for problem (1), under a more general assumption on the discontinuous leading coefficients $a_{i j}$, known as Chicco-condition, were proved in [7].

If $\Omega$ is an unbounded domain, problem (1) was studied, for instance, in [8], where the leading coefficients satisfy Cordes type condition and the lower order terms $a_{i}$, $a$ belong to suitable classes of Morrey type spaces.

It is well known that the primary tools in the proofs of existence and, sometimes, uniqueness of solution of elliptic boundary value problems of type (1) are $W^{2,2}$-a priori bounds. Our aim in this paper is to prove some weighted a priori estimates for a problem similar to (1) on unbounded domains of $\mathbb{R}^{n}, n \geq 2$, where the leading coefficients are in the class of discontinuity of Cordes type. More precisely, we consider the following Dirichlet problem:

$$
\left\{\begin{array}{l}
u \in W_{s}^{2,2}(\Omega) \cap \stackrel{\circ}{W}_{s}^{1,2}(\Omega), \\
L u=f, \quad f \in L_{s}^{2}(\Omega),
\end{array}\right.
$$

where $s \in \mathbb{R}, W_{s}^{2,2}(\Omega)$, $\stackrel{\circ}{W}_{s}^{1,2}(\Omega), L_{s}^{2}(\Omega)$ are weighted Sobolev spaces on an unbounded domain $\Omega$ of $\mathbb{R}^{n}, n \geq 2$, whose weight is a power of a function $\rho: \bar{\Omega} \rightarrow \mathbb{R}_{+}$of class $C^{2}(\bar{\Omega})$ such that

$$
\sup _{x \in \Omega} \frac{\left|\partial^{\alpha} \rho(x)\right|}{\rho(x)}<+\infty, \quad \forall|\alpha| \leq 2 .
$$

We recall that some $W_{s}^{2,2}$-a priori bounds as well as the well posedness of problem (4) were proved in [9] in the planar case, assuming that the matrix $A$ is uniformly elliptic, while the lower order terms satisfy hypotheses similar to those required in [8].

Here, we want to obtain the mentioned weighted a priori estimates in [9] also in the case $n>2$, under an assumption on the leading coefficients $a_{i j}$ of Cordes type and under hypotheses on the coefficients $a_{i}$ more general than those in [8].

The paper is organized as follows. In the next section we present basic notation and a class of Morrey type spaces where the lower order terms of our operator belong. In Section 3 we recall the definitions of our specific weight functions. Then we focus on certain classes of related weighted Sobolev spaces and we give auxiliary theorems to state our main results. In the last section we first establish some $W^{2,2}-$ a priori estimates and then, using a result in [10] related to the existence of a topological isomorphism from $W_{s}^{2,2}(\Omega)$ in $W^{2,2}(\Omega)$, we are finally able to derive our weighted $a$ priori bounds.

Taking into account the results of this paper, we are now in a position to approach the study of solvability of problem (4).

\section{Function spaces}

In this section we recall the definitions and some properties of a class of Morrey type spaces to which the lower order coefficients of our differential operator belong. These spaces of Morrey type are a generalization of the classical Morrey spaces $L^{p, \lambda}$ and strictly contain $L^{p, \lambda}\left(\mathbb{R}^{n}\right)$ when $\Omega=\mathbb{R}^{n}$ (see [11] and its bibliography).

Let us fix some notation. Let $G$ be a Lebesgue measurable subset of $\mathbb{R}^{n}$ and $\Sigma(G)$ be the $\sigma$-algebra of all Lebesgue measurable subsets of $G$. Given $F \in \Sigma(G)$ we denote by $|F|$ its 
Lebesgue measure and by $\chi_{F}$ its characteristic function. For every $x \in F$ and every $t \in \mathbb{R}_{+}$, we set $F(x, t)=F \cap B(x, t)$, where $B(x, t)$ is the open ball with center $x$ and radius $t$, and in particular we put $F(x)=F(x, 1)$.

The class of restrictions to $F$ of functions $\zeta \in C_{0}^{\infty}\left(\mathbb{R}^{n}\right)$ with $\bar{F} \cap \operatorname{supp} \zeta \subseteq F$ is denoted by $\mathfrak{D}(F)$ and, for $p \in\left[1,+\infty\left[, L_{\mathrm{loc}}^{p}(F)\right.\right.$ is the class of all functions $g: F \rightarrow \mathbb{R}$ such that $\zeta g \in L^{p}(F)$ for any $\zeta \in \mathfrak{D}(F)$.

From now on, $\Omega$ will be an unbounded open subset of $\mathbb{R}^{n}, n \geq 2$. A function $g \in L_{\text {loc }}^{p}(\bar{\Omega})$, $1 \leq p<+\infty$, belongs to the space of Morrey type $M^{p, \lambda}(\Omega), 0 \leq \lambda<n$, if the following norm is finite

$$
\|g\|_{M^{p, \lambda}(\Omega)}=\sup _{\substack{\tau \in] 0,1] \\ x \in \Omega}} \tau^{-\frac{\lambda}{p}}\|g\|_{L^{p}(\Omega(x, \tau)) .}
$$

We point out that

$$
M^{p_{0}, \lambda_{0}}(\Omega) \hookrightarrow M^{p, \lambda}(\Omega) \quad \text { if } p \leq p_{0} \text { and } \frac{\lambda-n}{p} \leq \frac{\lambda_{0}-n}{p_{0}}
$$

(see $[12,13]$ for details).

We denote by $\widetilde{M}^{p, \lambda}(\Omega)$ and $M_{o}^{p, \lambda}(\Omega)$ the closures of $L^{\infty}(\Omega)$ and $C_{0}^{\infty}(\Omega)$ in $M^{p, \lambda}(\Omega)$, respectively.

Furthermore, the following strict inclusion holds true:

$$
M_{o}^{p, \lambda}(\Omega) \subset \widetilde{M}^{p, \lambda}(\Omega)
$$

Let us put $M^{p}(\Omega)=M^{p, 0}(\Omega), \widetilde{M}^{p}(\Omega)=\widetilde{M}^{p, 0}(\Omega)$, and $M_{o}^{p}(\Omega)=M_{o}^{p, 0}(\Omega)$. For a general survey on Morrey type spaces $M^{p}(\Omega)$, we refer to [14].

In the end, we define the moduli of continuity of functions belonging to $\widetilde{M}^{p, \lambda}(\Omega)$ or $M_{o}^{p, \lambda}(\Omega)$. For $h \in \mathbb{R}_{+}$and $g \in M^{p, \lambda}(\Omega)$, we set

$$
F[g](h)=\sup _{\substack{A \in \Sigma(\Omega) \\ \sup _{x \in \Omega}|A(x)| \leq \frac{1}{h}}}\left\|g \chi_{A}\right\|_{M^{p, \lambda}(\Omega)} .
$$

As shown in [11], given $g \in M^{p, \lambda}(\Omega)$, the following characterizations hold:

$$
\begin{aligned}
& g \in \tilde{M}^{p, \lambda}(\Omega) \quad \Longleftrightarrow \lim _{h \rightarrow+\infty} F[g](h)=0, \\
& g \in M_{o}^{p, \lambda}(\Omega) \Longleftrightarrow \lim _{h \rightarrow+\infty}\left(F[g](h)+\left\|\left(1-\zeta_{h}\right) g\right\|_{M^{p, \lambda}(\Omega)}\right)=0,
\end{aligned}
$$

where $\zeta_{h}$, for any $h \in \mathbb{R}_{+}$, denotes a function of class $C_{0}^{\infty}\left(\mathbb{R}^{n}\right)$ such that

$$
0 \leq \zeta_{h} \leq 1, \quad \zeta_{h \mid \overline{B(0, h)}}=1, \quad \operatorname{supp} \zeta_{h} \subset B(0,2 h)
$$

Thus, if $g$ is a function in $\widetilde{M}^{p, \lambda}(\Omega)$, a modulus of continuity of $g$ in $\widetilde{M}^{p, \lambda}(\Omega)$ is a map $\tilde{\sigma}[g]$ : $\mathbb{R}_{+} \rightarrow \mathbb{R}_{+}$such that

$$
F[g](h) \leq \tilde{\sigma}[g](h), \quad \lim _{h \rightarrow+\infty} \tilde{\sigma}[g](h)=0 .
$$


While if $g$ belongs to $M_{o}^{p, \lambda}(\Omega)$, a modulus of continuity of $g$ in $M_{o}^{p, \lambda}(\Omega)$ is an application $\sigma_{o}[g]: \mathbb{R}_{+} \rightarrow \mathbb{R}_{+}$such that

$$
F[g](h)+\left\|\left(1-\zeta_{h}\right) g\right\|_{M^{p, \lambda}(\Omega)} \leq \sigma_{o}[g](h), \quad \lim _{h \rightarrow+\infty} \sigma_{o}[g](h)=0 .
$$

\section{Weight functions and weighted Sobolev spaces}

Next we recall some properties of a class of weights we are interested in, and we adapt to weighted framework some known embedding results.

Let $\Omega$ be an open subset of $\mathbb{R}^{n}$, not necessarily bounded, $n \geq 2$. Given $k \in \mathbb{N}_{0}$, we consider a weight function $\rho: \bar{\Omega} \rightarrow \mathbb{R}_{+}$such that $\rho \in C^{k}(\bar{\Omega})$ and

$$
\sup _{x \in \Omega} \frac{\left|\partial^{\alpha} \rho(x)\right|}{\rho(x)}<+\infty, \quad \forall|\alpha| \leq k
$$

As an example, we can think of the function

$$
\rho(x)=\left(1+|x|^{2}\right)^{t}, \quad t \in \mathbb{R} .
$$

From (10), by induction procedure, we can deduce the following property on the weight function $\rho$, shown in Lemma 2.1 of [10].

Lemma 3.1 If assumption (10) is satisfied, then

$$
\sup _{x \in \Omega} \frac{\left|\partial^{\alpha} \rho^{s}(x)\right|}{\rho^{s}(x)}<+\infty, \quad \forall s \in \mathbb{R}, \forall|\alpha| \leq k
$$

Some further interesting properties of the above weight functions can be found in [10]. Let us define now a class of weighted Sobolev spaces, with a weight function of the above-mentioned type. For $k \in \mathbb{N}_{0}, p \in[1,+\infty[, s \in \mathbb{R}$ and given a weight function $\rho$ satisfying (10), we denote by $W_{s}^{k, p}(\Omega)$ the space of distributions $u$ on $\Omega$ such that

$$
\|u\|_{W_{s}^{k, p}(\Omega)}=\sum_{|\alpha| \leq k}\left\|\rho^{s} \partial^{\alpha} u\right\|_{L^{p}(\Omega)}<+\infty
$$

equipped with the norm given in (12). Moreover, we put $W_{s}^{0, p}(\Omega)=L_{s}^{p}(\Omega)$ and we denote by $\mathscr{W}_{s}^{k, p}(\Omega)$ the closure of $C_{0}^{\infty}(\Omega)$ in $W_{s}^{k, p}(\Omega)$. A more detailed account of properties of weighted Sobolev spaces $W_{s}^{k, p}(\Omega)$ can be found in [10]. In particular, in Lemma 2.5 of [10], the authors proved the following result, which will be a fundamental tool in the proofs of our main results.

Lemma 3.2 Let $k \in \mathbb{N}_{0}, p \in[1,+\infty[$ and $s \in \mathbb{R}$. If assumption (10) is satisfied, then there exist two constants $c_{1}, c_{2} \in \mathbb{R}_{+}$such that

$$
c_{1}\|u\|_{W_{s}^{k, p}(\Omega)} \leq\left\|\rho^{t} u\right\|_{W_{s-t}^{k, p}(\Omega)} \leq c_{2}\|u\|_{W_{s}^{k, p}(\Omega)}, \quad \forall t \in \mathbb{R}, \forall u \in W_{s}^{k, p}(\Omega),
$$

with $c_{1}=c_{1}(t)$ and $c_{2}=c_{2}(t)$.

Moreover, if $\Omega$ has the segment property, then the map

$$
u \rightarrow \rho^{s} u
$$


defines a topological isomorphism from $W_{s}^{k, p}(\Omega)$ to $W^{k, p}(\Omega)$ and from $\stackrel{\circ}{W}_{s}^{k, p}(\Omega)$ to $\stackrel{\circ}{W}^{k, p}(\Omega)$.

For reader's convenience, we recall here an interpolation result which can be deduced from Theorem 3.1 of [15].

Theorem 3.3 If assumption (10) is satisfied for $k=2$, and $\Omega$ has the cone property, then for any $p \in\left[1,+\infty\left[, s \in \mathbb{R}\right.\right.$ and for any $u \in W_{s}^{2, p}(\Omega)$, we have $\partial^{\mu} u \in L_{s}^{p}(\Omega)$ for $|\mu|=1$. Moreover, there exists a constant $c \in \mathbb{R}_{+}$, depending on $\Omega$ and $p$, such that

$$
\left\|\partial^{\mu} u\right\|_{L_{s}^{p}(\Omega)} \leq c\left(\|u\|_{L_{s}^{p}(\Omega)}+\|u\|_{L_{S}^{p}(\Omega)}^{\frac{1}{2}}\left\|\partial^{2} u\right\|_{L_{s}^{p}(\Omega)}^{\frac{1}{2}}\right) .
$$

We end this section by proving some embedding results, adapted to our needs, which concern the boundedness of the multiplication operator

$$
u \rightarrow g u
$$

where the function $g$ belongs to suitable Morrey type spaces. Here Lemma 3.2 will play a crucial rule since it allows us to pass to no-weighted framework in order to exploit some well-known embedding estimates.

Theorem 3.4 Let $\Omega$ be an open subset of $\mathbb{R}^{n}$ having the cone property and assume (10) with $k=1$. If $g \in M^{r, \lambda}(\Omega)$, with $r>2$ and $\lambda=0$ if $n=2$, and $\left.\left.r \in\right] 2, n\right]$ and $\lambda=n-r$ if $n>2$, and $u \in W_{s}^{1,2}(\Omega)$, with $s \in \mathbb{R}$, then we have gu $\in L_{s}^{2}(\Omega)$. Moreover, there exists a constant $c \in \mathbb{R}_{+}$, depending only on $\Omega, n$, $r$ and $s$, such that

$$
\|g u\|_{L_{s}^{2}(\Omega)} \leq c\|g\|_{M^{r, \lambda}(\Omega)}\|u\|_{W_{s}^{1,2}(\Omega)} .
$$

Furthermore, if $g \in \widetilde{M}^{r, \lambda}(\Omega)$, then for any $\epsilon>0$ there exists a constant $c(\epsilon) \in \mathbb{R}_{+}$such that

$$
\|g u\|_{L_{s}^{2}(\Omega)} \leq \epsilon\|u\|_{W_{s}^{1,2}(\Omega)}+c(\epsilon)\|u\|_{L_{s}^{2}(\Omega)}, \quad \forall u \in W_{s}^{1,2}(\Omega),
$$

where $c(\epsilon)$ depends on $\epsilon, \Omega, n, r$, s and $\tilde{\sigma}[g]$.

If $g \in M_{o}^{r, \lambda}(\Omega)$, then for any $\epsilon>0$ there exist a constant $c^{\prime}(\epsilon) \in \mathbb{R}_{+}$and a bounded open subset $\Omega_{\epsilon} \subset \subset \Omega$ with the cone property such that

$$
\|g u\|_{L_{s}^{2}(\Omega)} \leq \epsilon\|u\|_{W_{s}^{1,2}(\Omega)}+c^{\prime}(\epsilon)\|u\|_{L^{2}\left(\Omega_{\epsilon}\right)}, \quad \forall u \in W_{s}^{1,2}(\Omega),
$$

where $c^{\prime}(\epsilon)$ and $\Omega_{\epsilon}$ depend on $\epsilon, \Omega, n, \rho, s, r$ and $\sigma_{o}[g]$.

Proof Let $u \in W_{s}^{1,2}(\Omega)$ and $g \in M^{r, \lambda}(\Omega)$. In view of Lemma 3.2 one has $\rho^{s} u \in W^{1,2}(\Omega)$. Hence, from Theorem 5.1 in [11] it follows that there exists a constant $c \in \mathbb{R}_{+}$such that

$$
\left\|\rho^{s} g u\right\|_{L^{2}(\Omega)} \leq c\|g\|_{M^{r, \lambda}(\Omega)}\left\|\rho^{s} u\right\|_{W^{1,2}(\Omega)},
$$

where $c$ depends on $\Omega, n, r$. Then, according to Lemma 3.2 and (19), we get (16). Now employing this embedding estimate we easily deduce (17) and (18). Indeed, fix $\epsilon>0$ and 
let $c$ be the constant in (16). If $g \in \widetilde{M}^{r, \lambda}(\Omega)$, then there exists $g_{\epsilon} \in L^{\infty}(\Omega)$ such that $\| g-$ $g_{\epsilon} \|_{M^{r, \lambda}(\Omega)}<\frac{\epsilon}{c}$. By $(16)$, for any $u \in W_{s}^{1,2}(\Omega)$, we get

$$
\|g u\|_{L_{S}^{2}(\Omega)} \leq c\left\|g-g_{\epsilon}\right\|_{M^{r, \lambda}(\Omega)}\|u\|_{W_{s}^{1,2}(\Omega)}+\left\|g_{\epsilon}\right\|_{L^{\infty}(\Omega)}\|u\|_{L_{S}^{2}(\Omega)} .
$$

Thus estimate (17) follows from (20).

On the other hand, if $g \in M_{o}^{r, \lambda}(\Omega)$, there exists $g_{\epsilon} \in C_{0}^{\infty}(\Omega)$ such that $\left\|g-g_{\epsilon}\right\|_{M^{r, \lambda}(\Omega)}<\frac{\epsilon}{c}$. Let $\Omega_{\epsilon}$ be a bounded open subset of $\Omega$, with the cone property, such that $\operatorname{supp} g_{\epsilon} \subset \Omega_{\epsilon}$. By (16) we have

$$
\begin{aligned}
\|g u\|_{L_{S}^{2}(\Omega)} & \leq c\left\|g-g_{\epsilon}\right\|_{M^{r, \lambda}(\Omega)}\|u\|_{W_{s}^{1,2}(\Omega)}+\left\|g_{\epsilon} u\right\|_{L_{s}^{2}\left(\Omega_{\epsilon}\right)} \\
& \leq \epsilon\|u\|_{W_{s}^{1,2}(\Omega)}+\left\|g_{\epsilon} \rho^{s}\right\|_{L^{\infty}\left(\Omega_{\epsilon}\right)}\|u\|_{L^{2}\left(\Omega_{\epsilon}\right)} .
\end{aligned}
$$

From this last inequality we get (18).

Theorem 3.5 Let $\Omega$ be an open subset of $\mathbb{R}^{n}$ having the cone property and let (10) be satisfied for $k=2$. If $g \in M^{t}(\Omega)$, with $t=2$ if $2 \leq n \leq 4, t>2$ if $n=4, t=n / 2$ if $n>4$, and $u \in W_{s}^{2,2}(\Omega)$, with $s \in \mathbb{R}$, then we have gu $\in L_{s}^{2}(\Omega)$. Moreover, there exists a constant $\in \mathbb{R}_{+}$, depending only on $\Omega, n$, $t$ and $s$, such that

$$
\|g u\|_{L_{s}^{2}(\Omega)} \leq c\|g\|_{M^{t}(\Omega)}\|u\|_{W_{s}^{2,2}(\Omega)} .
$$

Furthermore, if $g \in \tilde{M}^{t}(\Omega)$, then for any $\epsilon \in \mathbb{R}_{+}$there exists a constant $c(\epsilon) \in \mathbb{R}_{+}$such that

$$
\|g u\|_{L_{S}^{2}(\Omega)} \leq \epsilon\|u\|_{W_{s}^{2,2}(\Omega)}+c(\epsilon)\|u\|_{L_{s}^{2}(\Omega)}, \quad \forall u \in W_{s}^{2,2}(\Omega)
$$

where $c(\epsilon)$ depends on $\epsilon, \Omega, n, t$, s and $\tilde{\sigma}[g]$.

If $g \in M_{o}^{t}(\Omega)$, then for any $\epsilon>0$ there exist a constant $c^{\prime}(\epsilon) \in \mathbb{R}_{+}$and a bounded open subset $\Omega_{\epsilon} \subset \subset \Omega$ with the cone property such that

$$
\|g u\|_{L_{s}^{2}(\Omega)} \leq \epsilon\|u\|_{W_{s}^{2,2}(\Omega)}+c^{\prime}(\epsilon)\|u\|_{L^{2}\left(\Omega_{\epsilon}\right)}, \quad \forall u \in W_{s}^{2,2}(\Omega),
$$

where $c^{\prime}(\epsilon)$ and $\Omega_{\epsilon}$ depend on $\epsilon, \Omega, n, \rho, s, t$ and $\sigma_{o}[g]$.

Proof Assume $u \in W_{s}^{2,2}(\Omega)$ and $g \in M^{t}(\Omega)$. According to Lemma 3.2 we have $\rho^{s} u \in$ $W^{2,2}(\Omega)$. Thus, in view of Theorem 5.1 in [11], there exists a constant $c \in \mathbb{R}_{+}$such that

$$
\left\|\rho^{s} g u\right\|_{L^{2}(\Omega)} \leq c\|g\|_{M^{t}(\Omega)}\left\|\rho^{s} u\right\|_{W^{2,2}(\Omega)}
$$

where $c$ depends on $\Omega, n, t$. Therefore, Lemma 3.2 together with (25) give bound (22). Now, arguing as in Theorem 3.4, we easily deduce estimates (23) and (24).

\section{A priori estimates}

Our goal in this section is to give some $W_{s}^{2,2}$-a priori bounds for an elliptic second order linear differential operator of Cordes type. Here, the crucial analytic tools will be again Lemma 3.2 and certain unweighted a priori bounds, which we will prove at first. 
Let $\Omega$ be an unbounded open subset of $\mathbb{R}^{n}, n \geq 2$, such that:

$\left(\mathrm{h}_{0}\right) \quad \Omega$ has the uniform $C^{2}$-regularity property according to Adams [16].

Consider in $\Omega$ the second order linear differential operator in non-divergence form

$$
L=-\sum_{i, j=1}^{n} a_{i j} \frac{\partial^{2}}{\partial x_{i} \partial x_{j}}+\sum_{i=1}^{n} a_{i} \frac{\partial}{\partial x_{i}}+a .
$$

Concerning the leading coefficients, assume that

$\left(\mathrm{h}_{1}\right) \quad\left\{\begin{array}{l}a_{i j}=a_{j i} \in L^{\infty}(\Omega), \quad i, j=1, \ldots, n, \\ \operatorname{essinf}_{\Omega}\left(\sum_{i=1}^{n} a_{i i}\right)^{2}\left(\sum_{i, j=1}^{n} a_{i j}^{2}\right)^{-1}>n-1(\text { Cordes type condition })\end{array}\right.$

while for the lower order terms coefficients suppose

$$
\begin{aligned}
\left(\mathrm{h}_{2}^{\prime}\right) \quad a_{i} \in \tilde{M}^{r, \lambda}(\Omega), i=1, \ldots, n, \\
\\
\quad \text { with } r>2, \lambda=0 \text { if } n=2, \\
\quad \text { with } r \in] 2, n], \lambda=n-r \text { if } n>2, \\
\left(\mathrm{~h}_{3}^{\prime}\right) \quad a \in \tilde{M}^{t}(\Omega), \text { with } t=2 \text { if } 2 \leq n<4, t>2 \text { if } n=4, t=n / 2 \text { if } n>4 .
\end{aligned}
$$

Here we point out that the Cordes type condition, mentioned in hypothesis $\left(\mathrm{h}_{1}\right)$, entails that the operator $L$ defined in (26) is uniformly elliptic in $\Omega$. Moreover, it corresponds to uniform ellipticity if $n=2$.

We explicitly observe that, in view of Theorem 5.1 in [11], under the assumptions $\left(\mathrm{h}_{0}\right)$ $\left(\mathrm{h}_{3}^{\prime}\right)$, the operator

$$
L: W^{2,2}(\Omega) \rightarrow L^{2}(\Omega)
$$

is bounded.

We set

$$
L_{0}=-\sum_{i, j=1}^{n} a_{i j} \frac{\partial^{2}}{\partial x_{i} \partial x_{j}}
$$

Let us begin by providing an a priori bound of global type.

Lemma 4.1 Under hypotheses $\left(\mathrm{h}_{0}\right),\left(\mathrm{h}_{1}\right),\left(\mathrm{h}_{2}^{\prime}\right),\left(\mathrm{h}_{3}^{\prime}\right)$, there exists a constant $c \in \mathbb{R}_{+}$such that

$$
\|u\|_{W^{2,2}(\Omega)} \leq c\left(\|L u\|_{L^{2}(\Omega)}+\|u\|_{L^{2}(\Omega)}\right), \quad \forall u \in W^{2,2}(\Omega) \cap \stackrel{\circ}{W}^{1,2}(\Omega),
$$

where $c$ depends on $\Omega, n, r, t,\left\|a_{i j}\right\|_{L^{\infty}(\Omega)},\left\|a_{i}\right\|_{M^{r, \lambda}(\Omega)},\|a\|_{M^{t}(\Omega)}, \tilde{\sigma}\left[a_{i}\right]$ and $\tilde{\sigma}[a]$.

Proof Let $u \in W^{2,2}(\Omega) \cap \stackrel{\circ}{W}^{1,2}(\Omega)$. Employing (14) of Lemma 1 in [8] we get

$$
\left\|u_{x x}\right\|_{L^{2}(\Omega)} \leq c_{1}\left(\left\|L_{0} u\right\|_{L^{2}(\Omega)}+\|u\|_{L^{2}(\Omega)}\right),
$$


where $c_{1}$ depends on $\Omega, n,\left\|a_{i j}\right\|_{L^{\infty}(\Omega)}$. Thus taking into account (29), Corollary 5.2 in [11] and a well-known interpolation inequality (see Theorem 4.14 in [16]), we easily deduce estimate (28).

In order to prove another a priori bound of global type, we assume the following further assumptions on the coefficients of the operator $L$ :

$$
\begin{aligned}
& \left(\mathrm{h}_{2}\right) \quad \lim _{|x| \rightarrow+\infty} a_{i j}(x)=a_{i j}^{o}, \quad i, j=1, \ldots, n, \\
& \left(\mathrm{~h}_{3}\right) \quad\left\{\begin{array}{l}
a_{i} \in M_{o}^{r, \lambda}(\Omega), \\
a=a^{\prime}+b, \quad a^{\prime} \in M_{o}^{t}(\Omega), b \in L^{\infty}(\Omega), \operatorname{essinf}_{\Omega} b \geq b_{0}>0,
\end{array}\right.
\end{aligned}
$$

where the exponents $r, t$ and $\lambda$ are as in assumptions $\left(\mathrm{h}_{2}^{\prime}\right)$ and $\left(\mathrm{h}_{3}^{\prime}\right)$.

We observe that under hypotheses $\left(\mathrm{h}_{0}\right),\left(\mathrm{h}_{1}\right),\left(\mathrm{h}_{2}\right),\left(\mathrm{h}_{3}\right)$ the operator $(27)$ is also bounded, and the following a priori estimate holds.

Lemma 4.2 Under hypotheses $\left(\mathrm{h}_{0}\right),\left(\mathrm{h}_{1}\right),\left(\mathrm{h}_{2}\right),\left(\mathrm{h}_{3}\right)$, there exist a constant $c \in \mathbb{R}_{+}$and a bounded open subset $\Omega_{0}$ of $\Omega$ with the cone property such that

$$
\|u\|_{W^{2,2}(\Omega)} \leq c\left(\|L u\|_{L^{2}(\Omega)}+\|u\|_{L^{2}\left(\Omega_{0}\right)}\right), \quad \forall u \in W^{2,2}(\Omega) \cap \stackrel{\circ}{W}^{1,2}(\Omega),
$$

where $c$ and $\Omega_{0}$ depend on $\Omega, n, r, t,\left\|a_{i j}\right\|_{L^{\infty}(\Omega)},\left\|a_{i}\right\|_{M^{r, \lambda}(\Omega)},\|a\|_{M^{t}(\Omega)}, a_{i j}^{o}, \sigma_{o}\left[a_{i}\right], \sigma_{o}\left[a^{\prime}\right]$, $\|b\|_{L^{\infty}(\Omega)}$ and $b_{0}$.

Proof Assume $u \in W^{2,2}(\Omega) \cap \mathscr{W}^{1,2}(\Omega)$. According to Theorem 5 in [8] there exist $c_{1} \in \mathbb{R}_{+}$ and a bounded open subset $\Omega_{1} \subset \subset \Omega$ with the cone property such that

$$
\|u\|_{W^{2,2}(\Omega)} \leq c_{1}\left(\left\|L_{0} u+a u\right\|_{L^{2}(\Omega)}+\|u\|_{L^{2}\left(\Omega_{1}\right)}\right)
$$

where $c_{1}$ and $\Omega_{1}$ depend on $\Omega, n, t,\left\|a_{i j}\right\|_{L^{\infty}(\Omega)},\|a\|_{M^{t}(\Omega)}, a_{i j}^{o}, \sigma_{o}\left[a^{\prime}\right],\|b\|_{L^{\infty}(\Omega)}$ and $b_{0}$. Moreover, from Corollary 5.3 in [11] it follows that for any $\epsilon \in \mathbb{R}_{+}$there exist $c(\epsilon)$ and a bounded open subset $\Omega_{\epsilon} \subset \subset \Omega$ with the cone property such that

$$
\sum_{i=1}^{n}\left\|a_{i} u_{x}\right\|_{L^{2}(\Omega)} \leq \epsilon\|u\|_{W^{2,2}(\Omega)}+c(\epsilon)\left\|u_{x}\right\|_{L^{2}\left(\Omega_{\epsilon}\right)}
$$

where $c(\epsilon)$ and $\Omega_{\epsilon}$ depend on $\epsilon, \Omega, n, r,\left\|a_{i}\right\|_{M^{r, \lambda}(\Omega)}, \sigma_{o}\left[a_{i}\right]$.

Applying in (32) the classical interpolation inequality (see Theorem 4.14 in [16]) and using (31), we easily get bound (30).

Now we can use the estimates established above to obtain some $W_{s}^{2,2}$-a priori bounds. To this aim, from now on we consider a weight $\rho: \bar{\Omega} \rightarrow \mathbb{R}_{+}, \rho \in C^{2}(\bar{\Omega})$ and such that

$$
\left(\mathrm{h}_{4}\right) \quad \sup _{x \in \Omega} \frac{\left|\partial^{\alpha} \rho(x)\right|}{\rho(x)}<+\infty, \quad \forall|\alpha| \leq 2
$$


Let $L$ be the differential operator defined in (26). We explicitly observe that under assumptions $\left(\mathrm{h}_{0}\right),\left(\mathrm{h}_{1}\right),\left(\mathrm{h}_{2}^{\prime}\right),\left(\mathrm{h}_{3}^{\prime}\right)$ and $\left(\mathrm{h}_{4}\right)$, the operator

$$
L: W_{s}^{2,2}(\Omega) \rightarrow L_{s}^{2}(\Omega)
$$

is bounded, as consequence of Theorem 3.4 and Theorem 3.5.

Let us give the first weighted a priori estimate.

Theorem 4.3 Under hypotheses $\left(\mathrm{h}_{0}\right),\left(\mathrm{h}_{1}\right),\left(\mathrm{h}_{2}^{\prime}\right),\left(\mathrm{h}_{3}^{\prime}\right),\left(\mathrm{h}_{4}\right)$ and $s \in \mathbb{R}$, there exists a constant $c \in \mathbb{R}_{+}$such that

$$
\|u\|_{W_{s}^{2,2}(\Omega)} \leq c\left(\|L u\|_{L_{s}^{2}(\Omega)}+\|u\|_{L_{s}^{2}(\Omega)}\right), \quad \forall u \in W_{s}^{2,2}(\Omega) \cap \stackrel{\circ}{W}_{s}^{1,2}(\Omega),
$$

where $c$ depends on $\Omega, n, s, r, t,\left\|a_{i j}\right\|_{L^{\infty}(\Omega)},\left\|a_{i}\right\|_{M^{r, \lambda}(\Omega)},\|a\|_{M^{t}(\Omega)}, \tilde{\sigma}\left[a_{i}\right], \tilde{\sigma}[a]$.

Proof Fix $u \in W_{s}^{2,2}(\Omega) \cap \stackrel{\circ}{W}_{s}^{1,2}(\Omega)$. From Lemma 3.2 it follows that

$$
\rho^{s} u \in W^{2,2}(\Omega) \cap \mathscr{W}^{1,2}(\Omega) .
$$

Therefore, in view of Lemma 4.1, there exists $c_{1} \in \mathbb{R}_{+}$such that

$$
\left\|\rho^{s} u\right\|_{W^{2,2}(\Omega)} \leq c_{1}\left(\left\|L\left(\rho^{s} u\right)\right\|_{L^{2}(\Omega)}+\left\|\rho^{s} u\right\|_{L^{2}(\Omega)}\right)
$$

where $c_{1}$ depends on $\Omega, n, r, t,\left\|a_{i j}\right\|_{L^{\infty}(\Omega)},\left\|a_{i}\right\|_{M^{r, \lambda}(\Omega)},\|a\|_{M^{t}(\Omega)}, \tilde{\sigma}\left[a_{i}\right]$ and $\tilde{\sigma}[a]$.

Easy computations give

$$
\begin{aligned}
& \left\|\rho^{s} u\right\|_{W^{2,2}(\Omega)} \\
& \quad \leq c_{2}\left(\left\|\rho^{s} L u\right\|_{L^{2}(\Omega)}+\sum_{i=1}^{n}\left\|\rho^{s} u_{x_{i}}\right\|_{L^{2}(\Omega)}+\sum_{i=1}^{n}\left\|\rho^{s} a_{i} u\right\|_{L^{2}(\Omega)}+\left\|\rho^{s} u\right\|_{L^{2}(\Omega)}\right)
\end{aligned}
$$

where $c_{2} \in \mathbb{R}_{+}$depends on the same parameters as $c_{1}$ and on $s$.

Then, by Theorem 3.3, there exists a constant $c_{3} \in \mathbb{R}_{+}$depending only on $\Omega$ such that

$$
\sum_{i=1}^{n}\left\|u_{x_{i}}\right\|_{L_{S}^{2}(\Omega)} \leq c_{3}\left(\|u\|_{L_{S}^{2}(\Omega)}+\|u\|_{L_{S}^{2}(\Omega)}^{\frac{1}{2}}\left\|u_{x x}\right\|_{L_{S}^{2}(\Omega)}^{\frac{1}{2}}\right)
$$

On the other hand, from Theorem 3.4 it follows that for any $\epsilon \in \mathbb{R}_{+}$there exists $c_{1}(\epsilon) \in \mathbb{R}_{+}$ such that

$$
\sum_{i=1}^{n}\left\|a_{i} u\right\|_{L_{S}^{2}(\Omega)} \leq \epsilon\|u\|_{W_{s}^{1,2}(\Omega)}+c_{1}(\epsilon)\|u\|_{L_{S}^{2}(\Omega)},
$$

where $c_{1}(\epsilon)$ depends on $\epsilon, \Omega, n, r, s$ and $\tilde{\sigma}\left[a_{i}\right]$.

Hence, combining (36)-(38) with Lemma 3.2, we have

$$
\begin{aligned}
\|u\|_{W_{s}^{2,2}(\Omega)} \leq & c_{4}\left(\|L u\|_{L_{S}^{2}(\Omega)}+\|u\|_{L_{S}^{2}(\Omega)}+\epsilon\|u\|_{W_{s}^{2,2}(\Omega)}\right. \\
& \left.+c_{2}(\epsilon)\left(\|u\|_{L_{S}^{2}(\Omega)}+\|u\|_{L_{S}^{2}(\Omega)}^{\frac{1}{2}}\left\|u_{x x}\right\|_{L_{S}^{2}(\Omega)}^{\frac{1}{2}}\right)\right)
\end{aligned}
$$


where $c_{4}$ depends on the same parameters as $c_{2}$, and $c_{2}(\epsilon)$ depends on $\epsilon, \Omega, n, r, s$ and $\tilde{\sigma}\left[a_{i}\right]$.

If we choose $\epsilon=\frac{1}{2 c_{4}}$, by (39) we get

$$
\|u\|_{W_{S}^{2,2}(\Omega)} \leq c_{5}\left(\|L u\|_{L_{S}^{2}(\Omega)}+\|u\|_{L_{S}^{2}(\Omega)}+\|u\|_{L_{S}^{2}(\Omega)}^{\frac{1}{2}}\left\|u_{x x}\right\|_{L_{S}^{2}(\Omega)}^{\frac{1}{2}}\right)
$$

where $c_{5}$ depends on the same parameters of $c_{4}$.

Now, using Young's inequality in (40), we deduce (34).

In order to prove the second $W_{s}^{2,2}$-a priori bound, we need to add the following assumption on the weight function:

$$
\left(\mathrm{h}_{5}\right) \quad \lim _{|x| \rightarrow+\infty} \frac{\rho_{x}(x)+\rho_{x x}(x)}{\rho(x)}=0 .
$$

An example of a function verifying this hypothesis is given by

$$
\rho(x)=\left(1+|x|^{2}\right)^{t}, \quad t \in \mathbb{R} \backslash\{0\} .
$$

Theorem 4.4 Under hypotheses $\left(\mathrm{h}_{0}\right)-\left(\mathrm{h}_{5}\right)$, and $s \in \mathbb{R}$, there exist a constant $c \in \mathbb{R}_{+}$and a bounded open subset $\Omega_{1} \subset \subset \Omega$ with the cone property such that

$$
\|u\|_{W_{s}^{2,2}(\Omega)} \leq c\left(\|L u\|_{L_{s}^{2}(\Omega)}+\|u\|_{L^{2}\left(\Omega_{1}\right)}\right), \quad \forall u \in W_{s}^{2,2}(\Omega) \cap \stackrel{\circ}{W}_{s}^{1,2}(\Omega),
$$

where $c$ and $\Omega_{1}$ depend on $\Omega, n, s, r, t, a_{i j}^{o}, b_{0}, \rho,\left\|a_{i j}\right\|_{L^{\infty}(\Omega)},\left\|a_{i}\right\|_{M^{r, \lambda}(\Omega)},\|a\|_{M^{t}(\Omega)},\|b\|_{L^{\infty}(\Omega)}$, $\sigma_{o}\left[a_{i}\right]$ and $\sigma_{o}\left[a^{\prime}\right]$.

Proof Fix $u \in W_{s}^{2,2}(\Omega) \cap \stackrel{\circ}{W}_{s}^{1,2}(\Omega)$. Then by Lemma 3.2 we have

$$
\rho^{s} u \in W^{2,2}(\Omega) \cap \stackrel{\circ}{W}^{1,2}(\Omega) .
$$

Hence, in view of Lemma 4.2, there exist $c_{1} \in \mathbb{R}_{+}$and an open bounded subset $\Omega_{0} \subset \subset \Omega$ with the cone property such that

$$
\left\|\rho^{s} u\right\|_{W^{2,2}(\Omega)} \leq c_{1}\left(\left\|L\left(\rho^{s} u\right)\right\|_{L^{2}(\Omega)}+\left\|\rho^{s} u\right\|_{L^{2}\left(\Omega_{0}\right)}\right),
$$

where $c_{1}$ and $\Omega_{0}$ depend on $\Omega, n, r, t,\left\|a_{i j}\right\|_{L^{\infty}(\Omega)},\left\|a_{i}\right\|_{M^{r, \lambda}(\Omega)},\|a\|_{M^{t}(\Omega)}, a_{i j}^{o}, \sigma_{o}\left[a_{i}\right], \sigma_{o}\left[a^{\prime}\right]$, $\|b\|_{L^{\infty}(\Omega)}$ and $b_{0}$.

By (42), with simple calculations, we get the bound

$$
\begin{aligned}
\left\|\rho^{s} u\right\|_{W^{2,2}(\Omega)} \leq & c_{2}\left(\left\|\rho^{s} L u\right\|_{L^{2}(\Omega)}+\sum_{i, j=1}^{n}\left\|\rho^{s-2} \rho_{x_{i}} \rho_{x_{j}} u\right\|_{L^{2}(\Omega)}\right. \\
& +\sum_{i, j=1}^{n}\left\|\rho^{s-1} \rho_{x_{i}} u_{x_{j}}\right\|_{L^{2}(\Omega)}+\sum_{i, j=1}^{n}\left\|\rho^{s-1} \rho_{x_{i} x_{j}} u\right\|_{L^{2}(\Omega)} \\
& \left.+\sum_{i=1}^{n}\left\|\rho^{s} a_{i} u\right\|_{L^{2}(\Omega)}+\|u\|_{L^{2}\left(\Omega_{0}\right)}\right)
\end{aligned}
$$

where $c_{2}$ depends on the same parameters as $c_{1}$ and on $s, \rho$. 
Combining hypothesis $\left(\mathrm{h}_{5}\right)$ with statement (1.6) of [14], we obtain that the functions $\frac{\left|\rho_{x_{i}}\right|}{\rho} \cdot \frac{\left|\rho_{x_{j}}\right|}{\rho}, \frac{\left|\rho_{x_{i}}\right|}{\rho}$ and $\frac{\left|\rho_{x_{i} x_{j}}\right|}{\rho}$ belong to the space $M_{o}^{q}(\Omega)$ for any $q \in[1,+\infty[$. Therefore, from Theorem 3.5 it follows that for any $\epsilon \in \mathbb{R}_{+}$there exist $c_{1}(\epsilon), c_{2}(\epsilon), c_{3}(\epsilon) \in \mathbb{R}_{+}$and bounded open sets $\Omega_{1}(\epsilon), \Omega_{2}(\epsilon), \Omega_{3}(\epsilon) \subset \subset \Omega$ with the cone property such that

$$
\begin{aligned}
& \sum_{i, j=1}^{n}\left\|\frac{\rho_{x_{i}}}{\rho} \cdot \frac{\rho_{x_{j}}}{\rho} u\right\|_{L_{s}^{2}(\Omega)} \leq \epsilon\|u\|_{W_{s}^{2,2}(\Omega)}+c_{1}(\epsilon)\|u\|_{L^{2}\left(\Omega_{1}(\epsilon)\right)}, \\
& \sum_{i, j=1}^{n}\left\|\frac{\rho_{x_{i}}}{\rho} u_{x_{j}}\right\|_{L_{s}^{2}(\Omega)} \leq \epsilon\left\|u_{x}\right\|_{W_{s}^{1,2}(\Omega)}+c_{2}(\epsilon)\left\|u_{x}\right\|_{L^{2}\left(\Omega_{2}(\epsilon)\right)} \\
& \leq \epsilon\|u\|_{W_{s}^{2,2}(\Omega)}+c_{2}^{\prime}(\epsilon)\left(\|u\|_{L^{2}\left(\Omega_{2}(\epsilon)\right)}+\|u\|_{L^{2}\left(\Omega_{2}(\epsilon)\right)}^{\frac{1}{2}}\left\|u_{x x}\right\|_{L^{2}\left(\Omega_{2}(\epsilon)\right)}^{\frac{1}{2}}\right), \\
& \sum_{i, j=1}^{n}\left\|\frac{\rho_{x_{i} x_{j}}}{\rho} u\right\|_{L_{s}^{2}(\Omega)} \leq \epsilon\|u\|_{W_{s}^{2,2}(\Omega)}+c_{3}(\epsilon)\|u\|_{L^{2}\left(\Omega_{3}(\epsilon)\right)},
\end{aligned}
$$

where $c_{1}(\epsilon), c_{2}(\epsilon), c_{2}^{\prime}(\epsilon), c_{3}(\epsilon), \Omega_{1}(\epsilon), \Omega_{2}(\epsilon), \Omega_{3}(\epsilon)$ depend on $\epsilon, \Omega, n, \rho$ and $s$.

On the other hand, in view of Theorem 3.4, there exist $c_{4}(\epsilon)$ and a bounded open set $\Omega_{4}(\epsilon) \subset \subset \Omega$ with the cone property such that

$$
\sum_{i=1}^{n}\left\|a_{i} u\right\|_{L_{s}^{2}(\Omega)} \leq \epsilon\|u\|_{W_{s}^{1,2}(\Omega)}+c_{4}(\epsilon)\|u\|_{L^{2}\left(\Omega_{4}(\epsilon)\right)}
$$

where $c_{4}(\epsilon)$ and $\Omega_{4}(\epsilon)$ depend on $\epsilon, \Omega, n, \rho, s, r$ and $\sigma_{o}\left[a_{i}\right]$.

Bounds (43)-(47) together with Lemma 3.2 and Young's inequality give estimate (41).

\section{Competing interests}

The authors declare that they have no competing interests.

\section{Authors' contributions}

All authors contributed equally to the writing of this paper. All authors read and approved the final manuscript.

Received: 19 May 2015 Accepted: 14 July 2015 Published online: 30 July 2015

\section{References}

1. Gilbarg, D, Trudinger, NS: Elliptic Partial Differential Equations of Second Order, 2nd edn. Springer, Berlin (1983)

2. Maugeri, A, Palagachev, DK, Softova, LG: Elliptic and Parabolic Equations with Discontinuous Coefficients. Wiley, Berlin (2000)

3. Cordes, HO: Zero order a priori estimates for solutions of elliptic differential equations. Proc. Symp. Pure Math. 4, 157-166 (1961)

4. Talenti, G: Sopra una classe di equazioni ellittiche a coefficienti misurabili. Ann. Mat. Pura Appl. 69, 285-304 (1965)

5. Talenti, G: Equazioni lineari ellittiche in due variabili. Matematiche 21, 339-376 (1966)

6. Chicco, M: Equazioni ellittiche del secondo ordine di tipo Cordes con termini di ordine inferiore. Ann. Mat. Pura Appl. $85,347-356(1970)$

7. Chicco, M: Dirichlet problem for a class of linear second order elliptic partial differential equations with discontinuous coefficients. Ann. Mat. Pura Appl. 92, 13-23 (1972)

8. Transirico, M, Troisi, M: Equazioni ellittiche del secondo ordine di tipo Cordes in aperti non limitati di $\mathbb{R}^{n}$. Boll. Unione Mat. Ital. 3, 169-184 (1989)

9. D'Ambrosio, R, Sgambati, L, Transirico, M: Elliptic equations in weighted Sobolev spaces on unbounded domains of the plane. J. Anal. Appl. 8, 103-123 (2010)

10. Monsurrò, S, Salvato, M, Transirico, M: W2,2 A priori bounds for a class of elliptic operators. Int. J. Differ. Equ. (2011). doi:10.1155/2011/572824

11. Caso, L, D'Ambrosio, R, Monsurrò, S: Some remarks on spaces of Morrey type. Abstr. Appl. Anal. 2010, Article ID $242079(2010)$

12. Kufner, A, John, O, Fucík, S: Function Spaces. Noordhoff, Leyden (1977)

13. Piccinini, LC: Proprietà di inclusione e interpolazione tra spazi di Morrey e loro generalizzazione. Ann. Sc. Norm. Super. Pisa, Cl. Sci. (1969) 
14. Transirico, M, Troisi, M: Equazioni ellittiche del secondo ordine di tipo non variazionale in aperti non limitati. Ann. Mat. Pura Appl. 152, 209-226 (1988)

15. Glushak, AV, Transirico, M, Troisi, M: Teoremi di immersione ed equazioni ellittiche in aperti non limitati. Rend. Mat. 9, 113-130 (1989)

16. Adams, RA: Sobolev Spaces. Academic Press, New York (1975)

Submit your manuscript to a SpringerOpen ${ }^{\odot}$ journal and benefit from:

- Convenient online submission

- Rigorous peer review

- Immediate publication on acceptance

- Open access: articles freely available online

- High visibility within the field

- Retaining the copyright to your article

Submit your next manuscript at $\boldsymbol{~ s p r i n g e r o p e n . c o m ~}$ 